\title{
Egocentric and geocentric frames of reference in memory of large-scale space
}

\author{
TIMOTHY P. MCNAMARA and BJÖRN RUMP \\ Vanderbilt University, Nashville, Tennessee \\ and \\ STEFFEN WERNER \\ University of Idaho, Moscow, Idaho
}

\begin{abstract}
This experiment investigated the frames of reference used in memory to represent the spatial structure of a large-scale outdoor environment. Participants learned the locations of eight objects in an unfamiliar city park by walking through the park on one of two prescribed paths that encircled a large rectangular building. The aligned path was oriented with the building; the misaligned path was rotated by $45^{\circ}$. Later, participants pointed to target objects from imagined vantage points using their memories. Pointing accuracy was higher in the aligned than in the misaligned path group, and the patterns of results differed: In the aligned condition, accuracy was higher for imagined headings parallel to legs of the path and for an imagined heading oriented toward a nearby lake, a salient landmark. In the misaligned condition, pointing accuracy was highest for the imagined heading oriented toward the lake, and decreased monotonically with angular distance. These results indicated that locations of objects were mentally represented in terms of frames of reference defined by the environment but selected on the basis of egocentric experience.
\end{abstract}

Knowledge of the organization of objects in the environment is critical for many human activities. How is this knowledge represented in memory? In particular, what frames of reference are used by human memory systems to specify the spatial structure of the environment? In the first experiment of its kind, we had participants learn the locations of objects in a large city park by having them walk through the park on prescribed paths. Afterward, they pointed to target objects from imagined vantage points using their memories of the park. Our results indicate that locations of objects in a large-scale environment are mentally represented in terms of reference systems defined by the environment, and that these reference systems are selected using egocentric experience.

There are many ways to classify spatial reference systems (e.g., Levinson, 1996), but an especially useful one, for the purpose of understanding spatial memory and

Preparation of this article and the research reported in it were supported in part by National Institute of Mental Health Grant R01-MH57868, by Grant We 1973/3 from the German Science Foundation, Spatial Cognition Priority Program, and by the National Center for Geographic Information and Analysis Project Varenius. We are grateful to John Rieser for providing the equipment used to survey the park. Correspondence concerning this article should be addressed to T. P. McNamara, Department of Psychology, Vanderbilt University, 11121 st Avenue South, Nashville, TN 37203 (e-mail: t.mcnamara@vanderbilt.edu). navigation, draws a distinction between egocentric and geocentric (environmental) reference systems (e.g., Pani $\&$ Dupree, 1994). Egocentric reference systems specify location and orientation with respect to the organism and include eye, head, and body coordinates. Navigation by path integration (dead reckoning) is based on egocentric references systems. Geocentric reference systems define spatial relations with respect to features of the environment, such as the perceived direction of gravity, the Sun's azimuth, the Earth's magnetic field, and landmarks.

The use of both systems is well documented for several animal species (e.g., Etienne, 1992; Gallistel, 1990; Gothard, Skaggs, \& McNaughton, 1996; Wehner, Michel, $\&$ Antonsen, 1996). For example, honeybees and desert ants are continuously informed about their position relative to home via path integration, which is computed in part using self-induced retinal image flow (e.g., Müller \& Wehner, 1988; Ronacher \& Wehner, 1995; Srinivasan, Zhang, Altwein, \& Tautz, 2000). Shapes of landmarks are represented in retinotopic coordinates and recognized by image matching (e.g., Collett \& Cartwright, 1983; Wehner \& Räber, 1979). Geocentric frames of reference, such as Earth's magnetic field and the pattern of polarized light in the sky, are used to reduce error that would accumulate from purely egocentric sources of information (e.g., Collett \& Baron, 1994; Wehner et al., 1996). Remarkably little is known about the frames of reference used by nonhuman 
primates when navigating in natural environments, although electrophysiological studies have identified neurons in primate hippocampus corresponding to egocentric and to geocentric representations of space (e.g., Feigenbaum \& Rolls, 1991; Matsumura et al., 1999).

Egocentric and geocentric reference systems are also used by humans, although little is known about their relative importance in learning and remembering largescale outdoor environments. Humans use path integration but are much less efficient than honeybees or ants and probably do not depend on it when exploring unfamiliar territory over large distances (e.g., Loomis et al., 1993). The results of several studies indicate that egocentric reference systems are involved in the retrieval of knowledge about large-scale environments. Sholl (1987) required students at Boston College to point to campus landmarks from an indoor test site; participants were oriented but could not see the landmarks. Landmarks in front of observers were located more quickly than landmarks behind (see also Bryant \& Tversky, 1999; Franklin \& Tversky, 1990). In a similar study, Werner and Schmidt (1999) asked student residents of Göttingen, Germany, to imagine themselves at the intersection of two major streets in town, facing in various directions, and then to identify landmarks in cued directions. Landmarks in front of observers' imagined standing locations were identified faster and more accurately than landmarks behind. Because this effect occurred regardless of which direction participants imagined facing, it was probably caused by mechanisms involved in the retrieval of spatial knowledge or in the mapping of interobject directions onto an egocentric reference system for the purpose of making the response (Sholl \& Nolin, 1997).

Recent investigations have shown that the structure of an environment can affect the relative accessibility of remembered views of that environment and even influence whether or not an experienced view is mentally represented. Werner and Schmidt (1999) found that landmarks in Göttingen were identified faster and more accurately when the imagined heading was parallel to one of the major streets than when it was not (see also Montello, 1991). Shelton and McNamara (2001) examined the relative importance of egocentric and geocentric reference systems in memory of room-sized spaces. In Experiment 3 , objects were placed on a square mat that was oriented with the walls of the enclosing room (see Figure 1; real objects were used, not names). Participants learned the layout from two stationary points of view, one aligned and the other misaligned with the mat and the walls of the room. After learning the layout, participants made judgments of relative direction using their memories (e.g., "Imagine you are standing at the book and facing the wood. Point to the shoe."). Pointing accuracy (averaged across pointing direction) provided an objective measure of the difficulty of retrieving or inferring the spatial structure of the layout from various points of view. Participants were quite accurate pointing to objects from imagined views parallel to the aligned study view (e.g., at the book and facing the wood; at the

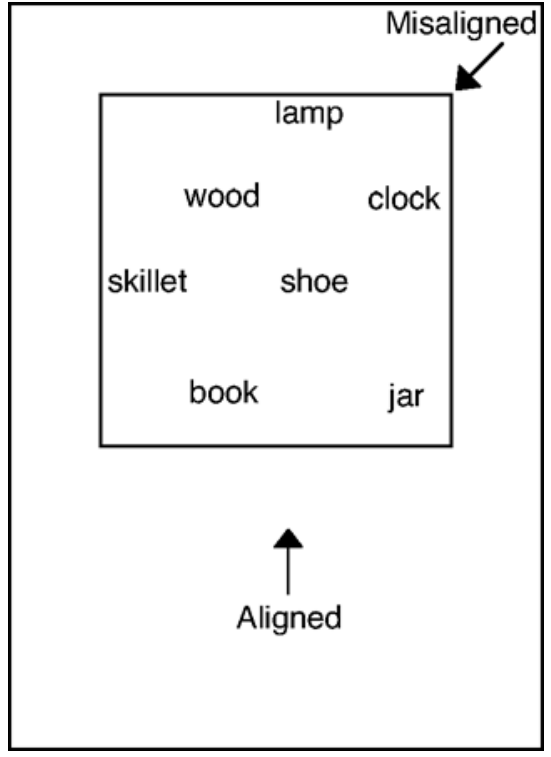

Figure 1. A schematic illustration of one of the layouts used by Shelton and McNamara (2001).

jar and facing the clock). However, they were no more accurate for imagined views parallel to the misaligned study view (e.g., at the lamp and facing the wood) than for imagined views parallel to unfamiliar headings (e.g., at the wood and facing the shoe). This pattern of results occurred even for participants who learned the misaligned view first. There was no behavioral evidence that participants had even seen the misaligned study view.

We have proposed a new theoretical framework to explain these findings (e.g., Mou \& McNamara, 2002; Shelton \& McNamara, 2001; Werner \& Schmidt, 1999). According to this theory, when people learn a new environment, they use various cues to select a geocentric reference system for encoding the spatial structure of that environment (e.g., Tversky, 1981). These cues include egocentric experience, the structure of the environment itself, and properties of the objects. Egocentric experience is the dominant cue because environments typically do not have inherently privileged directions or axes (e.g., humans cannot perceive magnetic fields). The act of interpreting the environment in terms of a spatial reference system is analogous to determining the "top" of a figure or an object (e.g., Rock, 1973); in effect, conceptual "north" is assigned to the layout, creating privileged directions in the environment (conceptual "north" need not, and usually will not, correspond to true or magnetic north or any other cardinal direction). The reference system that is selected determines the interpretation and hence the memory of the layout.

Consider, as an example, Shelton and McNamara's (2001, Experiment 3) results, described earlier. Observers who first learned the aligned view encoded the spatial structure of the layout in terms of a reference system aligned with their viewing perspective. Participants might 
have used, for example, the axes defined by the edges of the mat and the walls of the room. When participants moved to the misaligned viewpoint, they still interpreted the layout in terms of the reference system established at the aligned view, just as if they were viewing a (now) familiar object at a novel orientation. Hence, performance in judgments of relative direction was best for the heading parallel to the aligned view and was no better for the heading parallel to the misaligned view than for novel headings. Observers who first learned the misaligned view also must have interpreted the space in terms of a reference system defined by that view. This conclusion follows from the results of another experiment (Shelton \& McNamara, 2001, Experiment 2) in which participants learned the same layout but only from the misaligned point of view. Participants in this experiment were best able to retrieve the spatial structure of the layout from imagined views parallel to the misaligned view, and there was no evidence that they used the mat or the walls as reference systems. What happened to this mental representation of the layout when participants learned a second, aligned, view? Our hypothesis is that when participants were taken to the second, aligned, viewpoint, they reinterpreted the spatial structure of the layout in terms of a new reference system, one that was aligned with salient axes in the environment (e.g., the edges of the mat and the walls of the room) and with egocentric experience (albeit a new experience). After moving from a misaligned study view to an aligned study view, observers changed the definition of "north." A new spatial reference system-one that was aligned with the environment and egocentric experience-was selected and the spatial layout was reinterpreted in terms of it.

The present investigation had two related goals. The principal goal was to test our theoretical framework in a large-scale environment. Our previous experiments either examined preexperimentally acquired spatial memories, and therefore did not manipulate properties of the environment or participants' learning experiences (e.g., Werner \& Schmidt, 1999), or required observers to learn small-scale spaces from stationary viewing positions (e.g., Shelton \& McNamara, 2001). A second goal was to determine whether memories of large-scale spaces are orientation dependent or orientation independent. Although the preponderance of evidence indicates that memories of small-scale spaces are orientation dependent (i.e., certain perspectives, usually familiar ones, are better recognized and retrieved than are other perspectives), some findings indicate that memories of large-scale spaces may be orientation independent (e.g., Evans \& Pezdek, 1980; Richardson, Montello, \& Hegarty, 1999). In the present experiment, we required participants to learn the locations of objects in a large outdoor environment and manipulated the perspectives they were allowed to experience and the relations between those perspectives and salient elements of the landscape. Our goal was to assess the relative importance of, and possible interactions between, the experiences of the observer and the structure of the environment in the formation of spatial memories.

Participants were required to learn the locations of eight objects in a large city park. Two paths were used to dissociate the effects of egocentric experience and the structure of the environment. Both paths encircled the Parthenon (Figure 2), a full-scale replica of the Parthenon in Athens, Greece. The legs of the aligned path were parallel to the exterior walls of the Parthenon; the misaligned path was rotated by $45^{\circ}$ (Figure 3 ). The objects were located near the intersections of the two paths. After the learning phase, participants were taken to a remote site where they made judgments of relative direction using their memories of the park. Participants were required to imagine the environment from several vantage points and headings and to point to target objects (e.g., "Imagine you are standing at the tree and facing the condenser. Point to the car."). The angular error and latency of these judgments serve as objective measures

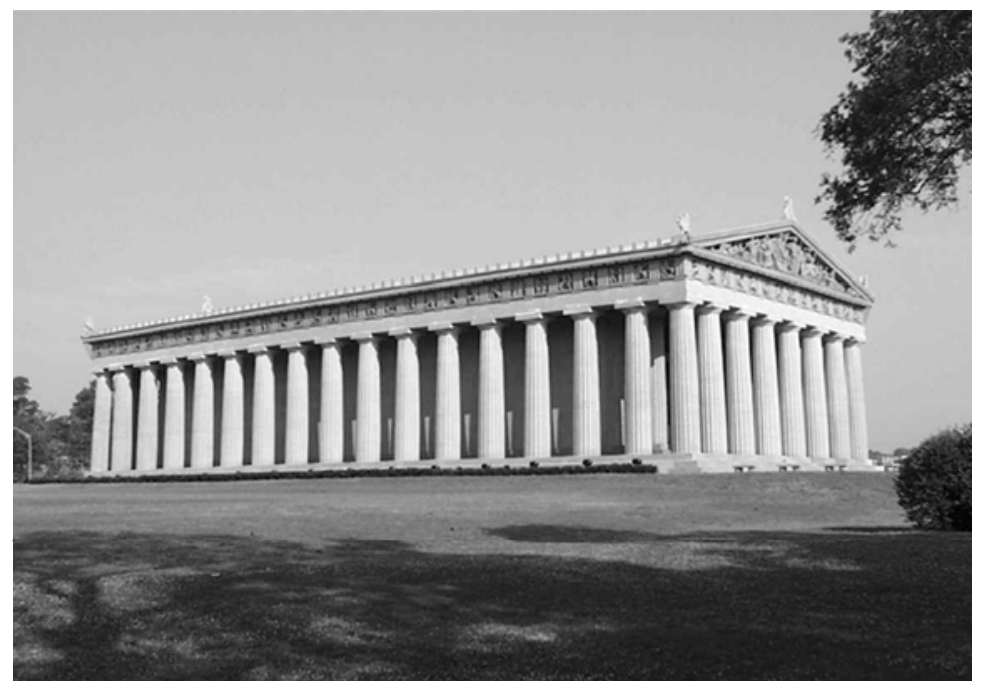

Figure 2. Parthenon, Centennial Park, Nash ville, Tennessee. 


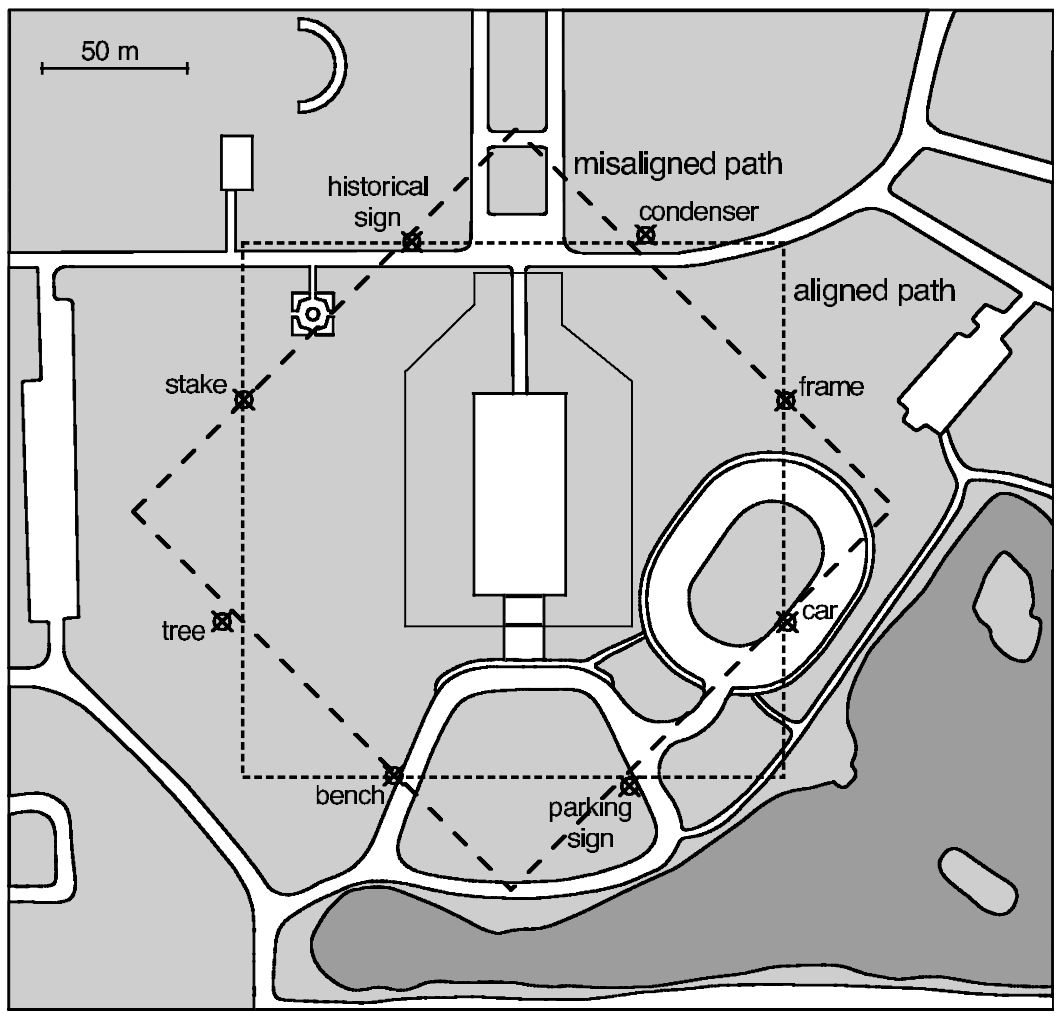

Figure 3. Map of the learning environment. Solid white rectangle in figure center is the Parthenon. Dashed lines indicate paths. Objects to be learned were located near intersections of the paths. Darker shaded area in lower right is the lake.

of the difficulty of retrieving or inferring the spatial structure of the layout from various points of view. We assume that spatial relations that are explicitly specified with respect to a particular spatial reference system can be retrieved from memory, whereas spatial relations that are not explicitly specified in terms of that spatial reference system must be inferred (e.g., Klatzky, 1998). These inferential processes produce measurable costs in terms of latency and error. Judgments based on retrieved spatial relations will therefore be faster and more accurate than judgments based on inferred spatial relations. Hence, we can use performance in judgments of relative direction as an index of the extent to which spatial relations were inferred, with poorer performance corresponding to a greater dependence on inferred, as opposed to retrieved, spatial relations. Similar conceptual analyses underlie the interpretation of data in related domains of inquiry, such as visual object recognition (e.g., Bülthoff, Edelman, \& Tarr, 1995).

We distinguished two prototypical patterns of results corresponding to the use of egocentric or geocentric frames of reference in representing the layout of objects. Each group experienced the park from four headings (aligned, $0^{\circ}, 90^{\circ}, 180^{\circ}, 270^{\circ}$; misaligned, $45^{\circ}, 135^{\circ}, 225^{\circ}$, $315^{\circ}$ ). If participants represented the park egocentrically, performance should be better on familiar headings than on unfamiliar headings (e.g., Diwadkar \& McNamara,
1997; Shelton \& McNamara, 1997); hence, patterns of performance should be identical in the two groups but differ in phase by $45^{\circ}$. Several patterns of results are consistent with the use of geocentric reference systems. To keep the exposition simple, we consider one. It is possible that the axes defined by the exterior walls of the Parthenon would be so salient that all participants would represent the layout of objects according to this geocentric reference system. In this case, performance in the two path groups would be identical, with better performance on headings of $0^{\circ}, 90^{\circ}, 180^{\circ}$, and $270^{\circ}$ than on other headings. We recognized that other elements of the park might be used as reference systems but anticipated that the Parthenon would be the most salient. To anticipate our results, the lake (see lower right corner of Figure 3) seems to have been at least as salient and influential as the Parthenon.

\section{METHOD}

\section{Participants}

Informed consent was obtained from 24 students (13 female) at Vanderbilt University. They received course credit for participating in the experiment.

\section{Design and Procedure}

Learning phase. The learning phase took part in Centennial Park, Nashville, Tennessee. Legs of the paths were $182 \mathrm{~m}$ in length. 
The paths were not visible; vertices were marked for the experimenter. The eight objects to be learned were near (maximum distance of $6.5 \mathrm{~m}$ ) the intersections of the two paths. Because of occlusion created by the Parthenon and other objects, the entire layout could not be viewed from any one vantage point.

Four groups were defined by the combination of path (aligned vs. misaligned) and direction of walking (clockwise vs. counterclockwise). Participants were randomly assigned to groups so that each group contained an approximately equal number of males and females. Only 1 participant had ever been in the park before the experiment.

Participants were blindfolded before entering the park to restrict their visual experience to the learning situation and to prevent them from knowing where they were with respect to the city. They were led to the corner of the appropriate path nearest the car (Figure 3) and the blindfold was removed. Participants were instructed to learn the locations of objects that were shown to them as they were guided along the path. They were told to keep track of the object locations as they walked but that they did not need to memorize other features of the park. The experimenter named the object and stopped for a few seconds as each was encountered. Participants were allowed to stop walking at any time to look around and to consider where objects were located, with the restriction that they had to maintain their current body orientation; they were allowed to turn their heads but not their bodies. After walking the path, participants were asked to name the objects in order of their occurrence. This circuit was completed twice and resulted in about 25 min of learning experience. All participants were highly familiar with the locations of the objects at the end of the learning phase.

Testing phase. Participants were transported by vehicle back to the laboratory for testing. The primary independent variable in judgments of relative direction was imagined heading. Each test trial was constructed from the names of three objects. Two objects established the imagined heading ("Imagine you are at the bench and facing the historical sign."); the third object was the target ("Point to the frame."). The eight headings parallel to legs of the paths were used $\left(0^{\circ}-315^{\circ}\right.$ in $45^{\circ}$ clockwise steps; $0^{\circ}$ was parallel to bench-historical sign). The 32 pairs of objects that produced these headings were each combined with three target objects, resulting in a total of 96 trials, 12 trials per heading. Target objects were selected to balance pointing direction across headings and the frequency of their occurrence. The configuration of objects allowed pointing directions from $23^{\circ}$ to $135^{\circ}$ and from $225^{\circ}$ to $315^{\circ}$ (defined egocentrically, so that $0^{\circ}=$ straight ahead).

Trials were presented on a Macintosh computer. Participants made their pointing judgments with an analogue joystick (Gravis Mac MouseStick II) resting on a countertop. Pointing accuracy and response latency were recorded, but the primary dependent measure was pointing accuracy. Feedback was not provided. Test trials were completed in approximately $40 \mathrm{~min}$.

\section{RESULTS}

Mean absolute angular error in pointing judgments is plotted in Figure 4 as a function of the path walked and imagined heading. Trials in which error exceeded $90^{\circ}$ or response time exceeded $60 \mathrm{sec}$ were excluded from analysis $(6.6 \%)$. Means were analyzed by way of analysis of variance with conditions of path (aligned vs. misaligned), direction of travel (clockwise vs. counterclockwise), and imagined heading $\left(0^{\circ}, 45^{\circ}, \ldots, 315^{\circ}\right)$. Path and direction of travel were between-participants variables. A separate analysis revealed no significant effects of gender.

As shown in Figure 4, there was an effect of imagined heading on accuracy of pointing to targets $[F(7,140)=$

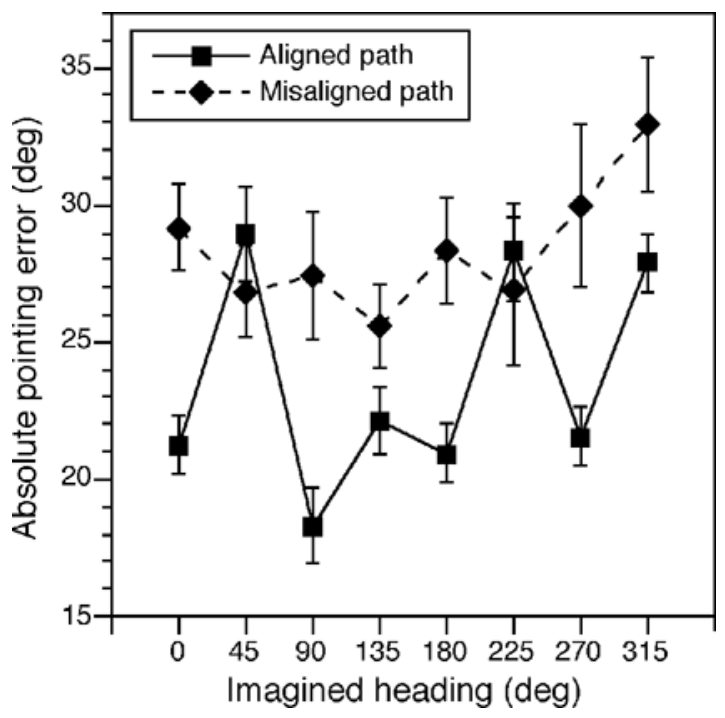

Figure 4. Mean absolute angular error in judgments of relative direction as a function of imagined heading and path traversed. (Error bars are confidence intervals corresponding to $\pm 1 S E M$ adjusted for between-participants variability.)

3.06, $\left.M S_{\mathrm{e}}=47.88, p=.005\right]$, but the pattern of results was different for the two groups $\left[F(7,140)=2.45, M S_{\mathrm{e}}=\right.$ $47.88, p=.021]$. In the aligned condition, pairwise comparisons showed that pointing error was equally low for the familiar headings of $0^{\circ}, 90^{\circ}, 180^{\circ}$, and $270^{\circ}$, and the unfamiliar heading of $135^{\circ}[t \mathrm{~s}(140) \leq 1.34, p \mathrm{~s} \geq .18]$, and significantly lower for these headings than for the remaining headings $[t \mathrm{~s}(140) \geq 2.05, p s \leq .042]$. Pointing error in the misaligned condition was lowest for the imagined heading of $135^{\circ}$ and increased monotonically with angular distance [quadratic contrast: $t(140)=2.54$, $p=.012]$.

Pointing error was lower in the aligned condition than in the misaligned condition $[F(1,20)=6.73, p=.017]$, indicating that the overall fidelity of spatial memory was higher in the aligned condition. Pairwise comparisons showed that performance in the two groups differed significantly for the headings of $0^{\circ}, 90^{\circ}, 180^{\circ}$, and $270^{\circ}$ $[t \mathrm{~s}(160) \geq 2.31, p \mathrm{~s} \leq .022]$ but not for the remaining headings $[t \mathrm{~s}(160) \leq 1.57, p \mathrm{~s} \geq .12]$; between-groups comparisons used the pooled error term $\left(M S_{\mathrm{e}}=61.93\right.$, $d f=160)$. There was no reliable effect of direction of travel $[F(1,20)<1]$.

Additional analyses in the aligned condition showed that views along legs of the path were retrieved more accurately than were views parallel to but not coincident with a path leg (e.g., "Imagine you are at the tree and facing the stake" vs. "Imagine you are at the bench and facing the historical sign"). Mean error was $18.3^{\circ}$ and $22.5^{\circ}$, respectively $[t(11)=3.13, p=.0096]$. This result suggests that spatial memories were both viewpoint and orientation dependent (e.g., Easton \& Sholl, 1995).

This experiment was not designed to examine effects of pointing direction. The layout did not permit pointing 
directions directly to the front or to the back or even unambiguously to the back (i.e., $135^{\circ}-225^{\circ}$ ). With this limitation in mind, we computed the mean pointing error for directions to the front and to the back of egocentric midline (excluding $90^{\circ}$ and $270^{\circ}$ ), collapsing across imagined heading. Pointing error was lower to the front than to the back $\left(\right.$ mean error $=24.5^{\circ}$ vs. $\left.28.0^{\circ}\right)[F(1,220)=$ 7.64, $p=.0062$ ], replicating prior results (e.g., Franklin \& Tversky, 1990; Sholl, 1987; Werner \& Schmidt, 1999).

Response latency revealed patterns similar to those in angular error; there was no evidence of speed-accuracy tradeoffs. The correlation between mean latency and mean angular error across imagined headings was .73 for the aligned path group and .67 for the misaligned path group.

\section{DISCUSSION}

To our knowledge, this experiment is the first investigation of spatial memory in which human participants have learned the locations of objects in an unfamiliar large-scale outdoor environment under conditions that controlled the views they were allowed to experience. Our results indicate that memories of large-scale spaces are not just collections of visual-spatial "snapshots" of experienced points of view. Participants in the aligned condition seem to have represented the layout from the unfamiliar heading of $135^{\circ}$, and those in the misaligned condition represented only one familiar orientation, not four, as purely egocentric coding would predict. Our results also show, however, that spatial memories are not determined solely by the physical structure of the environment; what matters is how that structure is experienced and interpreted. We wish to emphasize that all participants learned the locations of the same eight objects and were tested on the same judgments of relative direction. The striking differences in the accuracy with which various views of the environment could be retrieved or inferred must have been caused by the different paths traversed.

These results are consistent with the theoretical framework described in the introduction to this paper. According to this theory, learning the spatial structure of an unfamiliar environment involves interpreting it in terms of a spatial reference system. This spatial reference system is chosen using various cues, such as egocentric experience and properties of the environment itself. Our current conjecture (e.g., Mou \& McNamara, 2002) is that the spatial structure of the environment is represented in terms of an intrinsic reference system (e.g., Palmer, 1989), which is a type of geocentric reference system. In intrinsic reference systems, the location of an object is defined with respect to other objects in the layout (e.g., the rows and columns formed by chairs in a classroom). A collection of objects will have an infinite number of possible intrinsic axes, but because of perceptual grouping principles, such as proximity and similarity, some of these will be more salient than others.
Egocentric experience will also make some axes more salient than others.

Each of the paths corresponded to an alternative intrinsic frame of reference for representing the layout of objects. For example, the aligned path organizes the objects into "rows" defined by bench-parking sign, treecar, and so on, and "columns" defined by tree-stake, bench-historicalsign, and so on, whereas the misaligned path organizes the objects into "rows" defined by parking sign-car, bench-frame, and so on, and "columns" defined by bench-tree, parking sign-stake, and so on. We hypothesize that participants in the aligned condition represented the layout in terms of the intrinsic frame selected by their path and congruent with the Parthenon. Good performance for the unfamiliar heading of $135^{\circ}$ indicates that they also represented spatial relations in terms of the reference direction toward the lake, a very salient landmark. This direction corresponds to a "diagonal" in the intrinsic organization highlighted by the path and the Parthenon. Participants in the misaligned group seem to have represented the layout in terms of the single reference direction oriented toward the lake; this direction was one component of the intrinsic frame of reference highlighted by their path. There was no evidence that these participants represented the layout in terms of the axes parallel to the Parthenon. However, overall performance was worse in the misaligned than in the aligned group, indicating that the incongruity between the misaligned path and the axes defined by the Parthenon created difficulties in representing the spatial layout of the objects. Apparently, egocentric experience was not sufficient to cause participants in the misaligned group to represent the layout along the $45^{\circ}-225^{\circ}$ axis, or even in the $315^{\circ}$ direction (away from the lake). The lake might have been used by both groups because it is a salient geographical feature of the park and participants began their tours near its shore.

An important result of this experiment was that memories were orientation dependent. This finding agrees with the results of many investigations of memory of smallscale environments (for a review, see Shelton \& McNamara, 2001). We are aware of only one other experiment that has required participants to learn an unfamiliar largescale environment - albeit an indoor environment - and also tested the orientation dependence of the resulting memories. Richardson et al. (1999) required participants to learn the interior hallways of a large building by walking through the building (participants in other conditions learned the building by viewing a map or by navigating a desktop virtual environment). Afterward, participants engaged in several tasks, including pointing to target locations from imagined and actual locations in the building. Orientation dependence was tested by comparing pointing judgments for headings aligned with the first leg of the path to pointing judgments for other headings. Aligned judgments were no more accurate than misaligned judgments, suggesting that real movement in the building allowed participants to form orientation-independent men- 
tal representations. One concern we have with these results is that even in the best condition, performance was relatively poor (average pointing error of approximately $\left.40^{\circ}\right)$. It is possible that if alignment were defined with respect to a different reference axis (e.g., the longest leg of the path), or different reference axes for different participants (e.g., Valiquette, McNamara, \& Smith, 2003), evidence of orientation dependence might appear.

In conclusion, our results suggest that humans represent the spatial structure of large-scale environments in terms of reference systems defined by features of the environment. Spatial relations are thus not just sampled and stored as egocentric views from different vantage points but are structured according to geocentric reference systems. These geocentric reference systems, however, are selected on the basis of the views and paths experienced by the observer.

\section{REFERENCES}

Bryant, D. J., \& Tversky, B. (1999). Mental representations of perspective and spatial relations from diagrams and models. Journal of Experimental Psychology: Learning, Memory, \& Cognition, 25, 137156.

Bülthoff, H. H., Edelman, S. Y., \& TARr, M. J. (1995). How are threedimensional objects represented in the brain? Cerebral Cortex, $\mathbf{5}$, 247-260.

Collett, T. S., \& Baron, J. (1994). Biological compasses and the coordinate frame of landmark memories in honeybees. Nature, $\mathbf{3 6 8}$, 137-140.

Collett, T. S., \& Cartwright, B. A. (1983). Eidetic images in insects: Their role in navigation. Trends in Neurosciences, 6, 101-105.

Diwadkar, V. A., \& McNamara, T. P. (1997). Viewpoint dependence in scene recognition. Psychological Science, 8, 302-307.

Easton, R. D., \& Sholl, M. J. (1995). Object-array structure, frames of reference, and retrieval of spatial knowledge. Journal of Experimental Psychology: Learning, Memory, \& Cognition, 21, 483-500.

ETIENNE, A. S. (1992). Navigation of a small mammal by dead reckoning and local cues. Current Directions in Psychological Science, 1, 48-52.

Evans, G. W., \& Pezdek, K. (1980). Cognitive mapping: Knowledge of real-world distance and location information. Journal of Experimental Psychology: Human Learning \& Memory, 6, 13-24.

Feigenbaum, J. D., \& Rolls, E. T. (1991). Allocentric and egocentric spatial information processing in the hippocampal formation of the behaving primate. Psychobiology, 19, 21-40.

Franklin, N., \& Tversky, B. (1990). Searching imagined environments. Journal of Experimental Psychology: General, 119, 63-76.

Gallistel, C. R. (1990). The organization of learning. Cambridge, MA: MIT Press.

Gothard, K. M., Skaggs, W. E., \& McNaughton, B. L. (1996). Dynamics of mismatch correction in the hippocampal ensemble code for space: Interaction between path integration and environmental cues. Journal of Neuroscience, 16, 8027-8040.

KLATZKY, R. L. (1998). Allocentric and egocentric spatial representations: Definitions, distinctions, and interconnections. In C. Freksa, C. Habel, \& K. F. Wender (Eds.), Spatial cognition: An interdisciplinary approach to representing and processing spatial knowledge (pp. 1-17). New York: Springer-Verlag.
LeVinson, S. C. (1996). Frames of reference and Molyneaux's question: Crosslinguistic evidence. In P. Bloom, M. A. Peterson, L. Nadel, \& M. F. Garrett (Eds.), Language and space (pp. 109-169). Cambridge, MA: MIT Press.

Loomis, J. M., Klatzky, R. L., Golledge, R. G., Cicinelli, J. G., PelLEGRINO, J. W., \& FRY, P. A. (1993). Nonvisual navigation by blind and sighted: Assessment of path integration ability. Journal of Experimental Psychology: General, 122, 73-91.

Matsumura, N., Nishijo, H., Tamura, R, Eifuku, S., Endo, S., \& ONo, T. (1999). Spatial- and task-dependent neuronal responses during real and virtual translocation in the monkey hippocampal formation. Journal of Neuroscience, 19, 2381-2393.

Montello, D. R. (1991). Spatial orientation and the angularity of urban routes: A field study. Environment \& Behavior, 23, 47-69.

Mou, W., \& McNamara, T. P. (2002). Intrinsic frames of reference in spatial memory. Journal of Experimental Psychology: Learning, Memory, \& Cognition, 28, 162-170.

Müller, M., \& Wehner, R. (1988). Path integration in the desert ants, Cataglyphis fortis. Proceedings of the National Academy of Sciences USA, 85, 5287-5290.

Palmer, S. E. (1989). Reference frames in the perception of shape and orientation. In B. E. Shepp \& S. Ballesteros (Eds.), Object perception: Structure and process (pp. 121-163). Hillsdale, NJ: Erlbaum.

Pani, J. R., \& Dupree, D. (1994). Spatial reference systems in the comprehension of rotational motion. Perception, 23, 929-946.

Richardson, A. E., Montello, D. R. \& Hegarty, M. (1999). Spatial knowledge acquisition from maps and from navigation in real and virtual environments. Memory \& Cognition, 27, 741-750.

Rock, I. (1973). Orientation and form. New York: Academic Press.

Ronacher, B., \& Wehner, R. (1995). Desert ants Cataglyphis fortis use self-induced optic flow to measure distances travelled. Journal of Comparative Physiology A, 177, 21-27.

Shelton, A. L., \& McNamara, T. P. (1997). Multiple views of spatial memory. Psychonomic Bulletin \& Review, 4, 102-106.

Shelton, A. L., \& McNamara, T. P. (2001). Systems of spatial reference in human memory. Cognitive Psychology, 43, 274-310.

Sholl, M. J. (1987). Cognitive maps as orienting schemata. Journal of Experimental Psychology: Learning, Memory, \& Cognition, 13, 615628.

Sholl, M. J., \& Nolin, T. L. (1997). Orientation specificity in representations of place. Journal of Experimental Psychology: Learning, Memory, \& Cognition, 23, 1494-1507.

Srinivasan, M. V., Zhang, S., Altwein, M., \& Tautz, J. (2000). Honeybee navigation: Nature and calibration of the "odometer." Science, 287, 851-853.

Tversky, B. (1981). Distortions in memory for maps. Cognitive Psychology, 13, 407-433.

Valiquette, C. M., McNamara, T. P., \& Smith, K. (2003). Locomotion, incidental learning, and the selection of spatial reference systems. Memory \& Cognition, 31, 479-489.

Wehner, R., Michel, B., \& Antonsen, P. (1996). Visual navigation in insects: Coupling of egocentric and geocentric information. Journal of Experimental Biology, 199, 129-140.

WEHNER, R., \& RÄBER, F. (1979). Visual spatial memory in desert ants, Cataglyphis bicolor (Hymenoptera: Formicidae). Experientia, 35, 1569-1571.

Werner, S., \& SCHMidT, K. (1999). Environmental reference systems for large scale spaces. Spatial Cognition \& Computation, 1, 447-473.

(Manuscript received July 19, 2001; revision accepted for publication May 15, 2002.) 